\title{
Magnetomechanical damping by polycrystalline TbDy
}

\author{
N. Good and J. Dooley \\ Jet Propulsion Laboratory, 4800 Oak Grove Avenue, Pasadena, California 91109 \\ B. Fultz \\ Division of Engineering and Applied Science, California Institute of Technology, Pasadena, \\ California 91125
}

Vibration damping in polycrystalline TbDy alloys was studied at cryogenic temperatures. Mechanical hysteretic losses were measured at various strains, frequencies, and loading configurations at $77 \mathrm{~K}$. Some textured TbDy materials demonstrated $22.6 \%$ energy dissipation in mechanical measurements at low frequency $(0.01 \mathrm{~Hz})$ and a mean logarithmic decrement of 0.23 at a higher frequency $(25 \mathrm{kHz})$. Ultrasonic velocities of longitudinal and shear elastic waves were measured on single and polycrystalline TbDy; little variation in ultrasonic velocities was found even for samples with large variation in crystallographic texture and magnetomechanical properties. (C) 2002 American Institute of Physics. [DOI: 10.1063/1.1452217]

\section{INTRODUCTION}

Ferromagnetic TbDy alloys exhibit giant magnetostriction, approaching $1 \%$ at saturation. This large coupling between mechanical energy and magnetic energy enables the dissipation of vibration through the dynamics of magnetic domain reorientation. These processes are subject to hysteretic losses and result in mechanical damping. The damping depends on the magnetomechanical coupling factor describing the efficiency of converting elastic to magnetic energy, the magnetic anisotropy describing the difficulty of moment rotation, the Young's modulus, the magnetization, the magnetostriction, and the crystallographic texture. ${ }^{1}$ These properties and hence the damping characteristics of the material can be optimized through microstructural control. We expect the damping of optimized polycrystalline TbDy to be substantial based on its large magnetostriction, magnetomechanical coupling factors as high as $0.75,{ }^{2}$ and the relatively large hysteresis measured in curves of magnetostriction versus applied field. ${ }^{3-5}$

In previous work with polycrystalline TbDy, we measured quasi-static magnetomechanical energy dissipation as the ratio of the area of the stress-strain hysteresis loop to the mean area under the loading and unloading curves, $\Delta E / E$, at $\sim 0.01 \mathrm{~Hz} .{ }^{6}$ The energy lost is the product of the fractional dissipation with the total potential energy stored in the sample. The fraction of mechanical energy dissipated in the material was $19.2 \%$ over a range of $0-27 \mathrm{MPa}$ for commercial purity $\mathrm{TbDy}$ textured alloys at $77 \mathrm{~K}$.

\section{EXPERIMENT}

The TbDy alloys were prepared as drop-cast, cylindrical ingots which initially had a radial texture. The ingots were cold-rolled by $35 \%$ in thickness and annealed at $950{ }^{\circ} \mathrm{C}$ for $1.5 \mathrm{~h}$. The plane-rolling deformation is performed to reorient the grains such that the hard $c$-axes lie in the direction of applied deformation stress, and the annealing allows for strain relief and grain growth. A second cold-rolling deformation of $55 \%$ and heat treatment at $350{ }^{\circ} \mathrm{C}$ further reoriented the grains in the samples, which measured $9 \times 8$ $\times 4 \mathrm{~mm}$ (commercial-grade) and $17 \times 5 \times 4 \mathrm{~mm}$ (highpurity). The well-defined $c$-axis grain orientation was confirmed by thermal expansion analysis of the textured samples, an as-cast specimen and a single crystal TbDy control, the results of which are summarized in Table I. Specimens were prepared from commercial-grade TbDy with a typical purity of $99.7 \%$, with the main impurities being Ta, $\mathrm{O}$, and $\mathrm{N}$, and from high-purity $99.94 \%$ TbDy. Optical microscopy showed large variations of grain sizes and no clear differences between commercial and high-purity samples. The processed samples exhibited magnetostrictive strains of up to $56 \%$ of single crystal values. ${ }^{3-5}$

Ultrasonic measurements were made by affixing a piezoelectric transducer to the specimen, introducing a series of elastic pulses at $5 \mathrm{MHz}$, and measuring the round-trip sound wave propagation time with an oscilloscope. A screw-driven Instron load frame was operated in stroke control to apply compressive stresses to a prismatic bar of TbDy instru-

TABLE I. Thermal expansion analysis summary of polycrystalline and single crystal TbDy.

\begin{tabular}{lcccc}
\hline \hline Sample & $\begin{array}{c}x \text {-axis } \\
\text { expansion } \\
10^{-6} / \mathrm{K}\end{array}$ & $\begin{array}{c}y \text {-axis } \\
\text { expansion } \\
10^{-6} / \mathrm{K}\end{array}$ & $\begin{array}{c}z \text {-axis } \\
\text { expansion } \\
10^{-6} / \mathrm{K}\end{array}$ & $\begin{array}{c}x+y / \\
2 z\end{array}$ \\
\hline $\mathrm{Tb}_{60} \mathrm{Dy}_{40}$, single crystal & 3.3 & 3.3 & 14.7 & 0.22 \\
$\mathrm{~Tb}_{76} \mathrm{Dy}_{24}$, as-cast, $99.7 \%$ purity & 5.4 & 7.1 & 8.1 & 0.77 \\
$\mathrm{~Tb}_{76} \mathrm{Dy}_{24}$, textured, 99.94\% purity & 6.2 & 5.2 & 10.7 & 0.53 \\
$\mathrm{~Tb}_{76} \mathrm{Dy}_{24}$, textured, 99.7\% purity & 4.2 & 5.3 & 12.1 & 0.39 \\
\hline \hline
\end{tabular}


TABLE II. Fractional dissipation and Young's modulus of TbDy for various applied stress as at $77 \mathrm{~K}$.

\begin{tabular}{lcccc}
\hline \hline Sample & $\begin{array}{c}\text { Applied stress } \\
\text { range (MPa) }\end{array}$ & $\begin{array}{c}\text { Strain range } \\
(\mathrm{ppm})\end{array}$ & $\begin{array}{c}\text { Fractional } \\
\text { energy } \\
\text { dissipation }\end{array}$ & $\begin{array}{c}\text { Young's } \\
\text { modulus } \\
(\mathrm{GPa})\end{array}$ \\
\hline $\mathrm{Tb}_{76} \mathrm{Dy}_{24}$, & $0-36$ & $0-2150$ & $13.8 \%$ & $17.2^{\mathrm{a}}$ \\
polycrystalline & $0-14$ & $0-900$ & $16.7 \%$ & 15.6 \\
$99.94 \%$ purity & $14-28$ & $1100-1750$ & $12.9 \%$ & 21.5 \\
$\mathrm{~Tb}_{76} \mathrm{Dy}_{24}$, & $0-27$ & $0-2700$ & $19.2 \% \mathrm{a}$ & $10.0^{\mathrm{a}}$ \\
polycrystalline 99.7\% & $0-10$ & $0-900$ & $22.6 \%$ & 10.5 \\
purity & $10-20$ & $1250-2100$ & $16.5 \%$ & 13.3 \\
\hline \hline
\end{tabular}

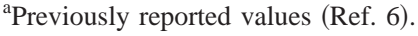

mented with electrical resistance strain gauges. Measurements were performed at room temperature and with the sample immersed in liquid nitrogen $\left(\mathrm{LN}_{2}\right)$. The stress-strain data showed magnetomechanical hysteresis when the sample was at $77 \mathrm{~K}$. High-frequency dynamic magnetomechanical damping measurements were made for comparison with the quasi-static results. The sample rested on an aluminum plate within a solenoid while immersed in $\mathrm{LN}_{2}$. A mechanical impulse was imparted to the sample and the induced current in the solenoid was measured.

\section{RESULTS}

Further analysis of the previously reported quasi-static minor loop stress-strain curves ${ }^{6}$ shows a number of interesting relationships summarized in Table II, with a typical TbDy stress-strain curve at $77 \mathrm{~K}$ and $300 \mathrm{~K}$, shown in Fig. 1. The commercial-grade specimen consistently showed larger strain amplitudes for the same applied stresses as compared to high-purity specimens. For the ranges measured, a 27\%$35 \%$ larger fractional energy dissipation was measured on commercial-grade specimens, with the highest ratio seen for the minor loops at low amplitudes. At low frequencies, $\Delta E / E$ of the minor loops varies inversely with the applied stress; for example, the commercial purity specimen shows a $\Delta E / E=22.6 \%$ over a range of 0-900 ppm, compared to $\Delta E / E=16.5 \%$ over a range of $1250-2100 \mathrm{ppm}$ - a differ- ence of $37 \%$. In general, minor loops at lower applied stresses exhibited lower Young's moduli as measured by the slopes of the stress-strain curves.

Measurements of ultrasonic velocities of shear and longitudinal waves in single crystal and textured TbDy samples at room temperature are summarized in Table III. While fairly large differences in magnetostrictive and magnetostrictive damping performance were seen between commercialgrade and high-purity samples of polycrystalline TbDy, the ultrasonic measurements revealed only small differences. Nevertheless, we expect a correlation between the measured ultrasonic velocities and the magnetization arising from an elastic wave traveling through the material. In addition, we include in Table III values of Young's modulus calculated from the ultrasonic velocities of the polycrystalline samples determined from the formula $Y=\rho V_{l}^{2}(1-\nu) /(1+\nu)(1$ $-2 \nu)$, with Poisson's ratio $\nu=\left(V_{l}^{2} / V_{t}^{2}-2\right) / 2\left(V_{l}^{2} / V_{t}^{2}-1\right)$ with $V_{l}$ and $V_{t}$ being the longitudinal and shear velocities, respectively. ${ }^{7}$ These results agree well with our measured room temperature Young's moduli in Table II.

Several aspects of the magnetoelastic behavior of the samples are apparent from the dynamical damping data. A typical result is shown in Fig. 2, with the induced voltage of the solenoid (proportional to current) plotted versus time. Since the magnetic sample is excited mechanically by an impact force, the decay of the inductive pickup can be attributed to the dissipation of vibrational energy within the

TABLE III. Ultrasonic velocities of shear and longitudinal waves in single crystal and polycrystalline TbDy at room temperature. Room temperature Young's moduli as measured by the slope of the stress-strain curve and derived from ultrasonic velocity measurements.

\begin{tabular}{|c|c|c|c|c|}
\hline \multirow[b]{2}{*}{ Sample } & \multicolumn{2}{|c|}{ Ultrasonic velocity $(\mathrm{km} / \mathrm{s})$} & \multirow{2}{*}{$\begin{array}{l}\text { Measured } \\
\text { Young's } \\
\text { modulus } \\
(\mathrm{GPa})\end{array}$} & \multirow{2}{*}{$\begin{array}{c}\text { Derived } \\
\text { Young's } \\
\text { modulus } \\
(\mathrm{GPa})\end{array}$} \\
\hline & Shear & Longitudinal & & \\
\hline $\begin{array}{l}\mathrm{Tb} \text {, single crystal } \\
c \text {-axis }\end{array}$ & 1.70 & 3.23 & & \\
\hline $\begin{array}{l}\text { Dy, single crystal, } \\
c \text {-axis }\end{array}$ & 1.71 & 3.09 & & \\
\hline $\begin{array}{l}\mathrm{Tb}_{60} \mathrm{Dy}_{40}, \text { single crystal, } \\
b \text {-axis }\end{array}$ & 1.64 & 2.89 & & \\
\hline $\begin{array}{l}\mathrm{Tb}_{76} \mathrm{Dy}_{24} \text {, polycrystalline } \\
99.7 \% \text { purity }\end{array}$ & 1.60 & 3.05 & 49.6 & 54.1 \\
\hline $\begin{array}{l}\mathrm{Tb}_{76} \mathrm{Dy}_{24} \text {, polycrystalline } \\
99.94 \% \text { purity }\end{array}$ & 1.60 & 3.00 & 57.2 & 55.2 \\
\hline
\end{tabular}




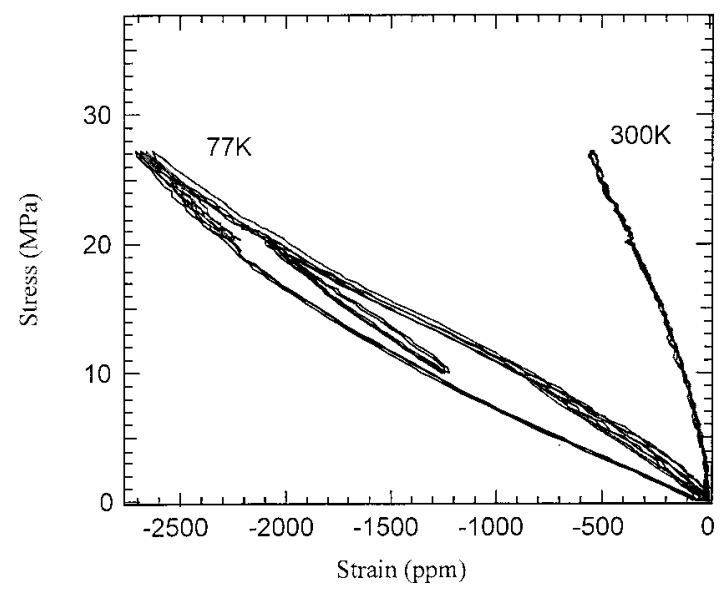

FIG. 1. Quasi-static magnetoelastic damping of $\mathrm{Tb}_{76} \mathrm{Dy}_{24}$ (99.7\% purity) at $77 \mathrm{~K}$ and $300 \mathrm{~K}$.

sample. The approximately $35-40 \mathrm{~ms}$ period corresponds to sound velocities of $1.6-1.8 \mathrm{~km} / \mathrm{s}$ across a specimen of 32.4 $\mathrm{mm}$. These are similar to measured sound velocities in polycrystalline TbDy of $1.7 \mathrm{~km} / \mathrm{s}$ and $2.9 \mathrm{~km} / \mathrm{s}$ for ultrasonic shear and longitudinal waves, respectively.

The corresponding mean logarithmic decrement $\delta$ $=\Delta E / 2 E=\ln \left(A_{n} / A_{n+1}\right)$ is often used to describe the damping capacity of materials, with $A_{n}$ corresponding to the vibrational amplitude of the $n$th oscillation of a vibrating element. By fitting the peaks of the oscillations in Fig. 2 to decaying exponential curves, we observe a decay in the amplitude of the signal peaks corresponding to $\delta=0.23$. This is within a



FIG. 2. Dynamic magnetoelastic damping signal from polycrystalline $\mathrm{Tb}_{60} \mathrm{Dy}_{40}$ at $77 \mathrm{~K}$. factor of 2 of our measured low-stress quasi-static damping capacities of TbDy alloys shown in Table II. This variation is not unexpected owing to the slightly different composition and a number of additional damping sources in the test system, for example, the interface of the specimen with the coil and the presence of external noise sources. Nevertheless, from these data the high-frequency damping capacity of TbDy is apparent.

\section{DISCUSSION}

At lower stresses, the magnetization proceeds by rearrangement of domain morphology, which is subject to losses associated with microstructural features. Perhaps the impurities in the commercial-grade material could be responsible for additional dissipation. Higher stresses can cause the magnetization to orient out of the basal plane along higherenergy directions, causing an effective stiffening of the material and a higher elastic modulus as shown in Table I.

Polycrystalline TbDy alloys with crystallographic texture were prepared, and their mechanical hysteresis was measured. An approximately $30 \%$ larger fractional energy dissipation was found for commercial-grade materials than for high-purity materials. At $25 \mathrm{kHz}$, a $\delta=0.23$ was measured for TbDy at $77 \mathrm{~K}$, comparable to the largest measured quasistatic fractional energy dissipation of $22.6 \%$. The high magnetomechanical damping capacities of textured TbDy polycrystalline alloys at low frequencies in quasi-static loadings and at higher frequencies in cyclic loading demonstrate the potential use of this material in cryogenic vibration damping applications.

\section{ACKNOWLEDGMENTS}

The authors thank Dr. A. E. Clark for stimulating discussions, Dr. M. Wun-Fogle for the use of several single crystal specimens, and Dr. T. Lograsso for preparing the ingots. The authors acknowledge support from NASA's Microgravity Division. The Jet Propulsion Laboratory is operated by the California Institute of Technology under contract with NASA.

${ }^{1}$ J. P. Teter, K. B. Hathaway, and A. E. Clark, J. Appl. Phys. 79, 6213 (1996).

${ }^{2}$ A. E. Clark, M. Wun-Fogle, J. B. Restorff, and J. F. Lindberg, IEEE Trans. Magn. 29, 3511 (1993).

${ }^{3}$ J. A. Dooley, C. A. Lindensmith, R. G. Chave, N. Good, J. Graetz, and B. Fultz, J. Appl. Phys. 85, 6256 (1999).

${ }^{4}$ R. Chave, C. Lindensmith, J. Dooley, B. Fultz, and M. Birsan, NASA Tech Briefs 23, 44 (1999).

${ }^{5}$ J. Dooley, N. Good, J. Graetz, R. Chave, and B. Fultz, Adv. Cryog. Eng. 46, 383 (1999).

${ }^{6}$ J. Graetz, N. Good, J. Dooley, R. Chave, and B. Fultz, J. Appl. Phys. 87, 5795 (2000)

${ }^{7}$ R. E. Green. Treatise on Materials Science and Technology, Vol. 3 (Academic, New York, 1973), p. 53. 